\title{
Imaging Mass Spectrometry with Silver Nanoparticles Reveals the Distribution of Fatty Acids in Mouse Retinal Sections
}

\author{
Takahiro Hayasaka, ${ }^{a}$ Naoko Goto-Inoue, ${ }^{a}$ Nobuhiro Zaima, ${ }^{a}$ \\ Kamlesh Shrivas, ${ }^{\mathrm{a}, \mathrm{b}}$ Yukiyasu Kashiwagi, ${ }^{\mathrm{c}}$ Mari Yamamoto, ${ }^{\mathrm{c}}$ \\ Masami Nakamoto, ${ }^{\mathrm{c}}$ and Mitsutoshi Setou ${ }^{\mathrm{a}}$ \\ ${ }^{a}$ Department of Molecular Anatomy, Molecular Imaging Frontier Research Center, Hamamatsu University \\ School of Medicine, Shizuoka, Japan \\ b Japan Society for the Promotion of Science (JSPS), Tokyo, Japan \\ c Osaka Municipal Technical Research Institute, Osaka, Japan
}

A new approach to the visualization of fatty acids in mouse liver and retinal samples has been developed using silver nanoparticles (AgNPs) in nanoparticle-assisted laser desorption/ ionization imaging mass spectrometry (nano-PALDI-IMS) in negative ion mode. So far, IMS analysis has concentrated on main cell components, such as cell membrane phospholipids and cytoskeletal peptides. AgNPs modified with alkylcarboxylate and alkylamine were used for nano-PALDI-IMS to identify fatty acids, such as stearic, oleic, linoleic, arachidonic, and eicosapentaenoic acids, as well as palmitic acid, in mouse liver sections; these fatty acids are not detected using 2,5-dihydroxybenzoic acid (DHB) as a matrix. The limit of detection for the determination of palmitic acid was $50 \mathrm{pmol}$ using nano-PALDI-IMS. The nano-PALDI-IMS method is successfully applied to the reconstruction of the ion images of fatty acids in mouse liver sections. We verified the detection of fatty acids in liver tissue sections of mice by analyzing standard lipid samples, which showed that fatty acids were from free fatty acids and dissociated fatty acids from lipids when irradiated with a laser. Additionally, we applied the proposed method to the identification of fatty acids in mouse retinal tissue sections, which enabled us to learn the six-zonal distribution of fatty acids in different layers of the retina. We believe that the current approach using AgNPs in nano-PALDI-IMS could lead to a new strategy to analyze basic biological mechanisms and several diseases through the distribution of fatty acids. (J Am Soc Mass Spectrom 2010, 21, 1446-1454) (c) 2010 American Society for Mass Spectrometry

I maging mass spectrometry (IMS) using conventional matrices, 2,5-dihydroxybenzoic acid (DHB), $2^{\prime}, 4^{\prime}, 6^{\prime}$-trihydroxyacetophenone (THAP), and pnitroaniline has been developed to reveal the distribution of biomolecules, such as phospholipids [1-4], proteins [5, 6], and glycolipids [7-9]. The advantages of IMS analysis are that it can visualize the distribution of biomolecules without labeling and identify the detailed structures of tissue sections. Traditional methods can also visualize the distribution of biomolecules, but those methods require antibodies and various fluorescent proteins. In the conventional IMS analysis, a tissue section sprayed with matrix solution is directly rasterscanned by matrix-assisted laser desorption/ionization (MALDI). The biomolecules in a tissue section are ionized by MALDI and separated by time-of-flight (TOF). The distribution of biomolecules is two-dimensionally visualized as relative signal intensities among measurement

Address reprint requests to Dr. T. Hayasaka, Department of Molecular Anatomy, Molecular Imaging Frontier Research Center, Hamamatsu University School of Medicine, 1-20-1 Handayama, Higashi-ku, Hamamatsu, Shizuoka 431-3192, Japan. E-mail: thaya@hama-med.ac.jp points on a tissue section. In this way, a great number of signals can be detected using a single IMS analysis. In a recent report, we demonstrated a technique to detect neutral lipids and lyso-phospholipids [3]. Many research groups have continued the development of the technique to detect various analytes.

Surface-assisted laser desorption/ionization (SALDI)MS using nanoparticles (NPs) has become a popular technique due to the rapid development of nanomaterials. SALDI-MS differs from MALDI-MS in several ways. For example, the mass spectra are simplified owing to a lack of chemical noise by the matrix [10]. The core metal and functional groups of the NPs can be modified to ionize analytes on the basis of specific chemical properties. The core metals gold [11], silver [12-14], titanium dioxide [15], and their mixture [16] have been used in SALDI-MS analysis. SALDI-MS analyses using their NPs have detected analytes that are different from those used in general matrices or to enhance the signal intensities from the molecules. The silver nanoparticles (AgNPs) enabled detection of glutathione [12], estrogens [13], cholesterol [14], and caro- 
tenoids [14] in SALDI-MS analyses. In a previous study, our research group reported that nanoparticle-assisted laser desorption/ionization (nano-PALDI)-IMS using iron oxide $\left(\mathrm{Fe}_{2} \mathrm{O}_{3}\right)$ as a core metal revealed the distributions of phosphatidylcholine (PC) (1-alk 38:3) and galactosylceramide (C24h:0) in rat cerebellum sections in positive ion mode [17]. The small diameter of $\mathrm{Fe}_{2} \mathrm{O}_{3} \mathrm{NPs}(3.7 \mathrm{~nm}$ ) enabled us to visualize the distribution of biomolecules with a high spatial resolution of 15 $\mu \mathrm{m}$ and then to distinguish among their complicated structures, such as the molecular layer, white matter, and granular layer, in rat cerebellum tissue. Moreover, nano-PALDI-IMS visualized the distribution of sulfatide and phosphatidylserine species in the rat hippocampus in negative ion mode [18]. Their lipid species were identified directly by MS/MS analyses.

The phospholipids in cell membrane contain many different fatty acids. The composition of fatty acids influences the development and maintenance of organ mechanisms in mammals. Long-chain polyunsaturated fatty acids (LCPUFAs), in particular arachidonic acid (AA; 20:4), eicosapentaenoic acid (EPA; 20:5), and docosahexaenoic acid (DHA; 22:6), are important structural components of membrane phospholipids and are required for normal development. Lipid and/or fatty acid composition in response to the intake or nutrients has been analyzed mainly in the liver [19], heart [19], endothelium [20], kidney [20], adipose tissue [21], red blood cells [22], plasma [19], brain [19], and retina [23]. Especially, DHA is the most interesting analyte in various organs. In the brain, the roles of DHA in development have been revealed. Careful studies in animals have shown that dietary n-3 fatty acid deficiency, such as DHA, reduces retinal and visual acuity [24]. The saturated fatty acids (SFA), such as palmitic acid (16:0) and stearic acid (18:0), also have important functions for impairing insulin sensitivity by reducing adiponectin secretion and impairing the insulin signaling pathways required for glucose uptake [25]. These results indicated that the synthesis and/or intake of nutritional sources rich in SFA and LCPUFAs influenced normal development and prevented several diseases. To resolve the roles of fatty acids in a biological object, it is very important to visualize their distributions. Yu et al. have also detected free fatty acids in positive-ion mode using MALDITOF-MS with meso-tetrakis porphyrin as matrix [26]. Cesium acetate was employed as a cationizing agent to form $[\mathrm{RCOOCs}+\mathrm{Cs}]^{+}$adduct. The use of cesium acetate instead of sodium acetate, which Ayorinde et al. [27] used as a dopant, significantly reduced the interfering peaks and facilitated the analysis of spectrum. The distributional information on fatty acids has been lost due to homogenization during sample preparation in analyses using gas and liquid chromatography.

In the present study, we tried to detect and visualize the fatty acids in biological tissue sections with nanoPALDI-IMS using AgNPs. AgNPs were coated on the surfaces of tissue sections of mice, and the fatty acids were identified by using nano-PALDI-IMS. The IMS system allowed for the detection of standard palmitic acid and indicated the limit of detection (LOD). The analysis of six standard lipid samples confirmed that their fatty acids were ionized after dissociation from lipids. The application of AgNPs for analysis of mouse retinas supported our previous study that the PC composed of DHA was strongly distributed around the pigment epithelium. This is the first finding of the specific detection and visualization of fatty acids in biological sections in nanoPALDI-IMS analyses using AgNPs.

\section{Experimental}

\section{Chemicals}

AgNPs were synthesized by using the described proto$\mathrm{col}$ in the literature [28]. The AgNPs were prepared from the precursors of $n-\mathrm{C}_{13} \mathrm{H}_{27} \mathrm{COOAg}$ and stearylamine, and their mean diameter was $3.84 \pm 0.45 \mathrm{~nm}$. We observed the AgNPs by a transmission electron microscope (TEM) and measured the diameter of AgNPs from the TEM images. The DHB matrix was purchased from Bruker Daltonics (Bremen, Germany). Human angiotensin II and human bradykinin fragment 1-7 were purchased from Sigma Aldrich (St. Louis, MO, USA). The standard lipids, such as PC (16:0/18:1), phosphatidylserine [PS (18:0/18:1)], phosphatidylinositol [PI (18:1/18:1)], sphingomyelin [SM (d18:1/16:0)], cardiolipin [CA (18:1/18:1/18:1/18: 1)], and phosphoinositol-3,5-bisphosphate [ $\mathrm{PIP}_{2}(18: 0 /$ 20:4)], were purchased from Avanti Polar Lipids (Alabaster, AL, USA). The standard fatty acid of palmitic acid was also purchased from Avanti Polar Lipids. Hexane, methanol, and ultra-pure water were purchased from Wako Pure Chemical (Osaka, Japan). All the chemicals used in this study were of the highest purity available.

\section{Sample Preparation for LOD Determination in nano-PALDI-IMS}

Palmitic acid (16:0) was prepared in concentrations of 1 $\mathrm{mM}, 100 \mu \mathrm{M}$, and $10 \mu \mathrm{M}$ in $70 \%$ methanol. A $0.5 \mu \mathrm{L}$ solution was deposited on the well of the MS target plate, and thus the final volumes of the droplets were 500, 50, and 5 pmol. A thin-layer of AgNPs was applied as a matrix to the surface by using a $0.2-\mathrm{mm}$ nozzle caliber airbrush (Procon Boy FWA Platinum; Mr. Hobby, Tokyo, Japan). Spraying with $500 \mu \mathrm{L}$ of AgNPs solution $(50 \mathrm{mg} / \mathrm{mL}$ in $100 \%$ hexane) was performed, and the solutions were allowed to dry at room temperature. During spraying, the distance between the nozzle and the tissue surface was kept at $15 \mathrm{~cm}$. The droplets of palmitic acid were measured by nano-PALDI-IMS analyses. The mass spectra were acquired from each measurement point and were normalized. The ion images at $\mathrm{m} / \mathrm{z} 255.4$ were reconstructed by flexImaging (Bruker Daltonics). To determine the LOD for palmitic acid on the MS target plate, the region of interest (ROI) was selected from each ion image by 35 points. The mass 
spectra in the ROIs were combined with a script program. The LOD was determined from the value of signal-to-noise ratio $(\mathrm{S} / \mathrm{N})$. The $\mathrm{S} / \mathrm{N}$ ratio value was acquired by calculating the ratio using the signal intensity of the peak at $m / z 255.3$ and the maximum signal intensity within $10 \mathrm{u}$ around the peak at $m / z$ 255.3. To indicate the method reproducibility in this experiment, the signal intensities were collected. The significant difference against the signal intensity with the $P$ value of 0.01 on blank, only AgNPs, was analyzed by the Student's $t$-test.

\section{Sample Preparations for Detecting Fatty Acids in Standard Lipids in nano-PALDI-IMS}

The six standard lipids-SM (d18:1/16:0), PC (16:0/18: 1), PS (18:0/18:1), CA (18:1/18:1/18:1/18:1), PI (18:1/ $18: 1)$, and $\mathrm{PIP}_{2}(18: 0 / 20: 4)$ - were prepared to the concentration of $1 \mathrm{mM}$ in $100 \% \mathrm{MeOH}$, and $1 \mu \mathrm{L}$ solutions were deposited on the wells of MS target plates by a pipetter. The total amount of each lipid was $1 \mathrm{nmol}$. The droplets were coated by spraying $500 \mu \mathrm{L}$ of AgNPs solution using an airbrush and measured by IMS analysis with a scan pitch of $300 \mu \mathrm{m}$. The peaks observed at $m / z 255.3$ (16:0), $m / z 281.3$ (18:1), and $m / z 283.3$ (18:0) were selected to reconstruct the ion images of the fatty acids.

\section{Sample Preparation for Analyses of Mouse Liver and Retinal Sections in nano-PALDI-IMS}

The care and use of laboratory animals were in accordance with the Animal Experiment Regulations of Hamamatsu University School of Medicine, which follow the Guidelines for the Proper Conduct of Animal Experiments by the Science Council of Japan. Eight-wkold male C57BL/6J mice were purchased from Japan SLC (Shizuoka, Japan). The mice were euthanized, and liver and retina samples were isolated immediately. The mouse organs were frozen in liquid nitrogen and stored at $-80^{\circ} \mathrm{C}$. Before sectioning, the frozen samples were kept for $30 \mathrm{~min}$ at $-18^{\circ} \mathrm{C}$ (liver) and $-28^{\circ} \mathrm{C}$ (retina). The serial sections were prepared to compare the different of peaks detected by spraying AgNPs or DHB matrix solution $(50 \mathrm{mg} / \mathrm{mL} \mathrm{DHB}$ in $70 \%$ methanol/ $0.1 \%$ TFA). The sections were sliced to a thickness of 10 $\mu \mathrm{m}$ using a cryostat (CM 1950; Leica Microsystems, Wetzlar, Germany). Sections were thaw-mounted onto an indium-tin-oxide (ITO)-coated glass slide (Bruker Daltonics) and dried at room temperature. The sections were placed in a polycarbonate tube and stored at $-20{ }^{\circ} \mathrm{C}$ until IMS analysis. An optimal cutting temperature compound (Sakura Finetek, Torrance, CA) was used only to fix frozen samples onto the support stand of the cryostat; it was not used for embedding the frozen samples, because the residual polymer on the sections might have degraded the mass spectra. Before IMS analysis, the sections stored in tubes were kept at room temperature for $20 \mathrm{~min}$. A thin AgNPs or DHB matrix layer was applied to the surface by an airbrush. After each tissue section was sprayed with $500 \mu \mathrm{L}$ of AgNPs or DHB matrix solution, an ITO-coated glass slide was attached to an MTP Slide Adapter II (Bruker Daltonics). IMS analyses were performed with a scan pitch of $100 \mu \mathrm{m}$ (liver) and $10 \mu \mathrm{m}$ (retina).

\section{IMS Operating Conditions}

All IMS analyses were performed by a MALDI-TOF/ TOF-type mass spectrometer (Ultraflex II; Bruker Daltonics) in negative-ion and reflector modes. The TOF/ TOF analyzer was calibrated by mono-DHB $\left([\mathrm{M}-\mathrm{H}]^{-}\right.$, $m / z$ 153.03), human bradykinin fragment 1-7 ([M $\mathrm{H}]^{-}, m / z$ 755.40), and human angiotensin II $\left([\mathrm{M}-\mathrm{H}]^{-}\right.$, $m / z$ 1044.54). The laser was run at $200 \mathrm{~Hz}$, and the mass spectra were obtained from 200 shots of Nd:YAG laser per measurement point. The flexControl settings, such as laser energy, detector gain, and random-walk function, were optimized to maximize the sensitivity of the signal detection for each analysis. The areas of IMS analyses were selected from the optical image by flexImaging. The obtained mass spectra were normalized and all ion images were reconstructed by flexImaging. To compare the mass spectra obtained on measurement areas, the ROI was selected on an ion image and the mass spectra were combined using the script program in flexAnalysis provided by Bruker Daltonics.

\section{Results and Discussion}

\section{LOD Determination of Palmitic Acid in nano-PALDI-IMS}

In this experiment, we tried to detect a fatty acid by IMS analysis in negative-ion mode. For this purpose, three different volumes of palmitic acid (16:0) were deposited on each well of the MS target plate. In the results of IMS analysis, the monoisotopic peak at $\mathrm{m} / \mathrm{z} 255.3$ and the isotope series peaks corresponding to palmitic acid were confirmed in the mass spectra obtained on the droplet of palmitic acid (Figure 1a-c). There are many large peaks except for the peak at $m / z 255.3$ in the mass spectra, such as the peak at $m / z$ 227.3. The peaks were also observed in mass spectrum on background (Figure 1d). Therefore, the peaks are neither fragments nor decomposition from the palmitic acid. In this experiment, the palmitic acid was detected in nano-PALDIIMS using AgNPs as shown in Figure 1.

The LOD is a very important parameter for validating the proposed method in biological applications. The LOD value was determined at a signal-to-noise $(\mathrm{S} / \mathrm{N})$ ratio of 3 . The value of 3 has been used to evaluate the LOD in another study [16]. The peaks at $m / z 255.3$ corresponding to palmitic acid are indicated by arrows. The $\mathrm{S} / \mathrm{N}$ ratios were acquired by calculating the ratio using the signal intensity of the peak at $m / z 255.3$ and the maximum signal intensity within $10 \mathrm{u}$ around the peak at $m / z$ 255.3. The $S / N$ ratios obtained were 70.3, 
(a)

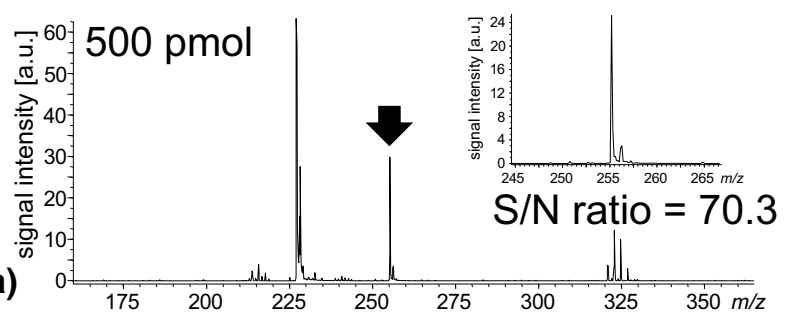

(b)

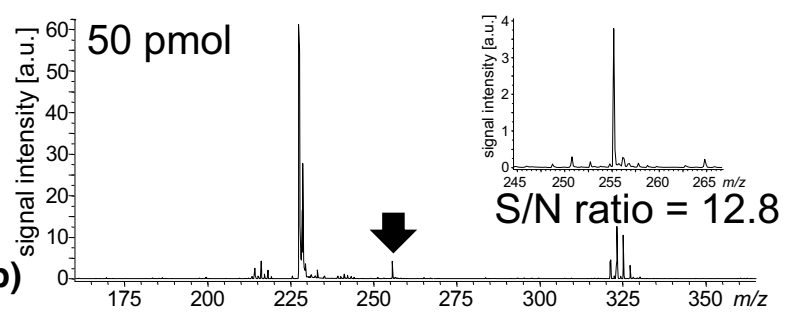

(c)

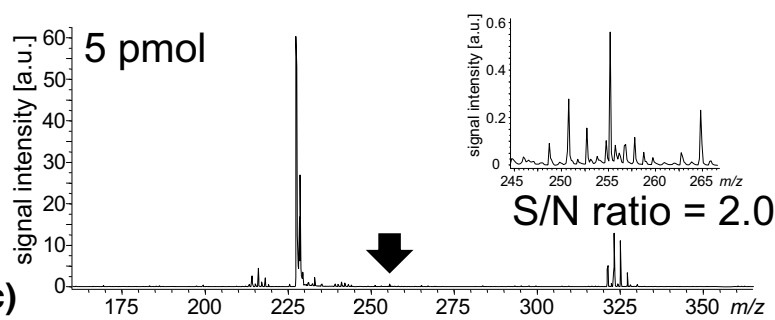

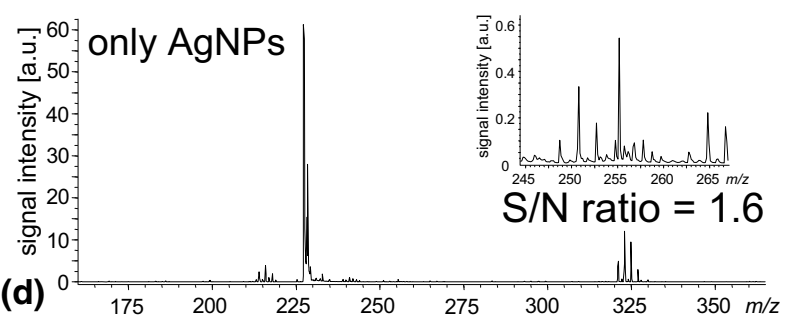
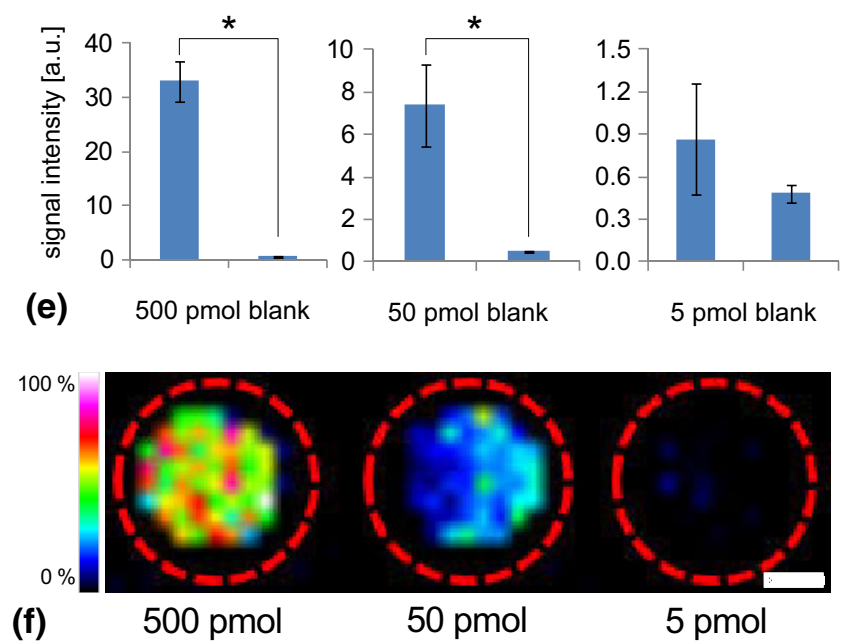

Figure 1. LOD determination of palmitic acid in nano-PALDI-IMS. Three different volumes of palmitic acid were deposited on each well of the target plate and AgNPs solution was sprayed on the surface. The surfaces were measured with a scan pitch of $300 \mu \mathrm{m}$ by nano-PALDI-IMS analysis using AgNPs in negative-ion mode. The positions of each well are circled with red dot lines. The scale bar is $1 \mathrm{~mm}$. The mass spectra from the ROI are shown. The $\mathrm{S} / \mathrm{N}$ ratios are 70.3, 12.8, and 2.0 for 500 (a), 50 (b), and 5 pmol (c), respectively. The mass spectrum of background is also shown (d). The peaks at $\mathrm{m} / \mathrm{z} 255.4$ are indicated by arrows. Values of bar graphs are represented as mean \pm standard deviation $(n=6)(\mathbf{e}) .{ }^{*} P<0.01$. The ion image of palmitic acid at $m / z 255.4$ was reconstructed (f).

12.8, and 2.0 for 500 pmol (Figure 1a), 50 pmol (Figure $1 \mathrm{~b}$ ), and 5 pmol (Figure 1c), respectively. Therefore, the LOD obtained by using nano-PALDI-IMS was 50 pmol. To indicate the method reproducibility in this experiment, the signal intensities were collected and statistically analyzed using the Student's $t$-test (Figure 1e). The signal intensities acquired on the amount of 500 and 50 pmol were significantly larger than that on blank. Moreover, all standard deviations with the low value demonstrated the method reproducibility.

The ion images at $\mathrm{m} / \mathrm{z} 255.3$ corresponding to palmitic acid were reconstructed by the use of flexImaging software (Figure 1f). The ion images of palmitic acid were obtained at 500 and $50 \mathrm{pmol}$, and showed that we were able to detect the molecules at both amounts. However, we were unable to reconstruct any image at 5 pmol of palmitic acid. We found that the use of AgNPs for nano-PALDI-IMS enabled us to detect fatty acids such as palmitic acid.

\section{Identification of Fatty Acids from Mouse Liver Sections in nano-PALDI-IMS}

For IMS analysis, liver sections are good samples to confirm, through ion images, whether or not the ion signals could be detected, because the structure is almost homogeneous in all regions of tissue samples.
The nano-PALDI-IMS method was applied to identify the fatty acids from the mouse liver sections. AgNPs or DHB matrix solution was sprayed onto the serial sections of mouse liver, and the results of IMS analyses were compared. The mass spectra were obtained by IMS analyses using AgNPs or DHB matrix in negativeion mode. The tendency of the mass spectrum in nano-PALDI-IMS using AgNPs is different from that in MALDI-IMS using the DHB matrix. Many peaks in the range of $\mathrm{m} / \mathrm{z} 200-350$ (Figure 2a) were detected on tissue section when AgNPs were used as a matrix in nanoPALDI-IMS. The peak at $m / z 227.3$ was detected as a major signal for the analysis of liver samples, as we saw during the LOD determination of palmitic acid in nano-PALDIIMS (Figure 1). The peak was also detected on background, only AgNPs (Figure 3b). Therefore, we believe that the peak at $m / z 227.3$ is associated with AgNPs. On the other hand, the mass spectrum obtained by using DHB as a matrix in MALDI-IMS showed a little of the peaks in the mass range of $m / z 200-350$. The peaks are considered as biomolecules on mouse liver by the comparison with the mass spectrum on background of DHB (Figure 2d) and did not correspond to fatty acids.

Six ion images were reconstructed from liver samples using AgNPs and DHB matrix in IMS (Figure 2e). As indicated in mass spectra acquired in nano-PALDI- 

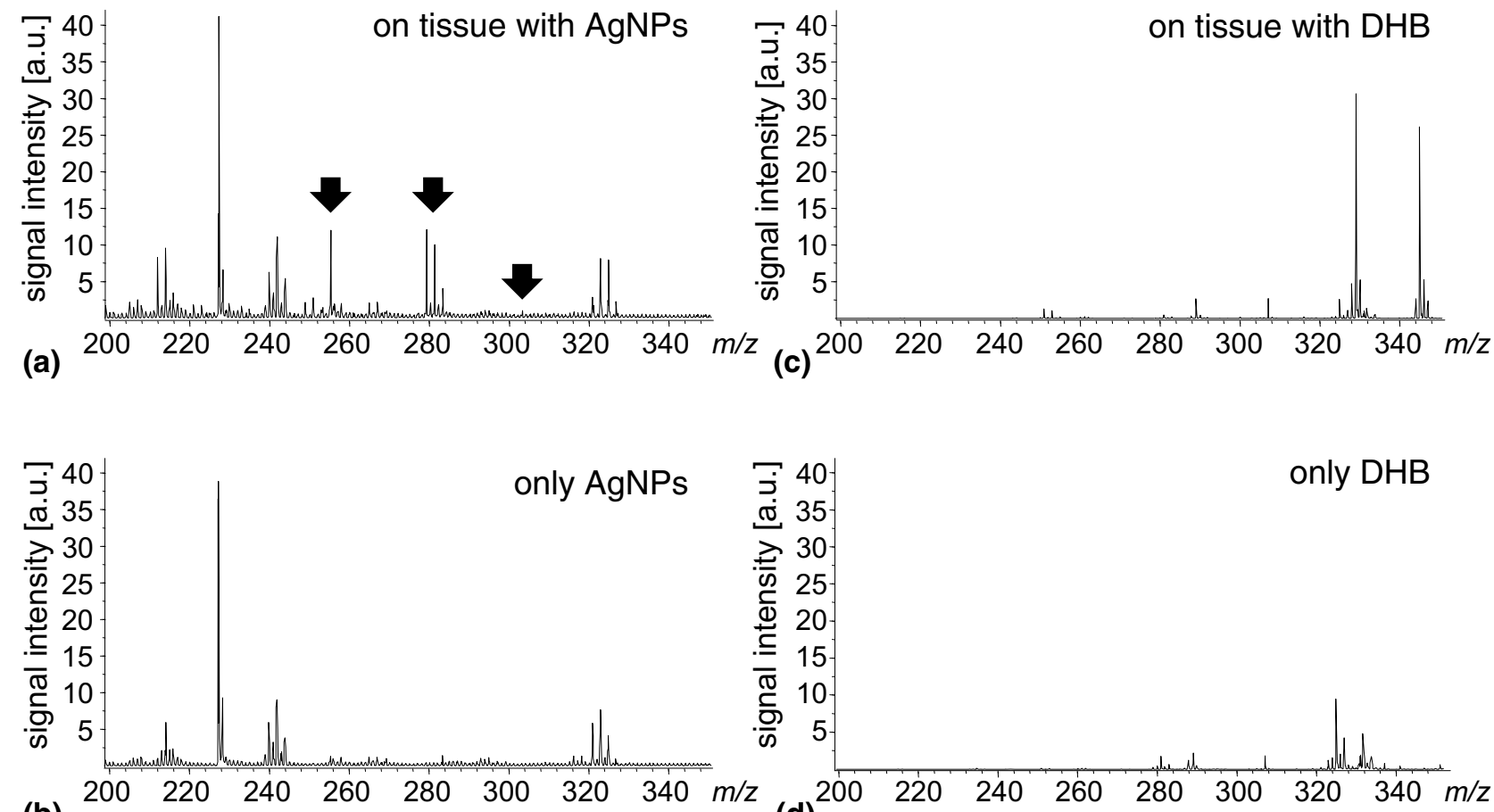

(b)
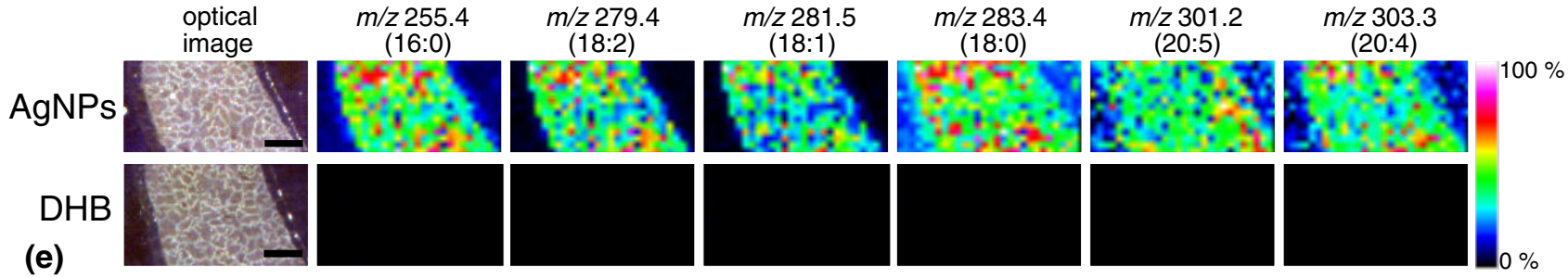

Figure 2. Identification of fatty acids from mouse liver sections in nano-PALDI-IMS. The serial sections were sliced to a thickness of $10 \mu \mathrm{m}$. AgNPs or DHB matrix solution was sprayed on the surface of the mouse liver sections, respectively. Their sections were measured with a scan pitch of 100 $\mu \mathrm{m}$ by nano-PALDI-IMS analysis in negative-ion mode. The mass spectra were obtained from the sections sprayed AgNPs (a) on tissue section, (b) only AgNPs or DHB matrix (c) on tissue section, (d) only DHB solution. The peaks used to reconstruct ion image were indicated by arrows. In the analysis using AgNPs and DHB, the ion signals at $m / z 255.4$ (16:0), 279.4 (18:2), 281.5 (18:1), 283.4 (18:0), 301.2 (20:5), and 303.3 (20:4) were detected (e). The scale bars are $500 \mu \mathrm{m}$.

IMS using AgNPs, the ion images were visualized at lower mass range in mouse liver sections at $\mathrm{m} / \mathrm{z}$ 255.4, 279.4, 281.5, 283.4, 301.2, and 303.3, which corresponded to palmitic acid (16:0), linoleic acid (18:2), oleic acid (18:1), stearic acid (18:0), EPA (20:5), and AA (20:4), respectively (Figure 2e). On the other hand, in MALDIIMS using DHB matrix, there were no signals. Thus, the nano-PALDI-IMS with the use of AgNPs enabled us to visualize the distributions of fatty acids in mouse liver samples. However, it remained unclear whether the ion signals referred to the dissociation from lipids or to free fatty acids from the liver samples.

\section{Identification of Fatty Acids from Standard Lipids in nano-PALDI-IMS}

It is very important to reveal whether the ion signals of fatty acids referred to the dissociation from lipids or only free fatty acids in biological sections to which AgNPs were applied in nano-PALDI-MS. To resolve this question, six standard lipids were prepared. The standard lipids were deposited in their respective wells of the MS target plate: SM (d18:1/16:0) on the no. 1 well, PC (16:0/18:1) on the no. 2 well, PS (18:0/18:1) on the no. 3 well, CA $(18: 1 / 18: 1 / 18: 1 / 18: 1)$ on the no. 4 well, PI (18:1/18:1) on the no. 5 well, and PIP $_{2}$ (18:0/20:4) on the no. 6 well. The samples deposited on the target plate were measured by nano-PALDI-IMS analysis by using AgNPs. The representative mass spectra acquired on each well are shown in Figure 3a-f. The arrows indicate the peaks used to reconstruct ion images of three fatty acids, palmitic acid (16:0, Figure 3g), oleic acid (18:1, Figure $3 \mathrm{~h}$ ), and stearic acid (18:0, Figure 3i). The ion image in each well is indicated by numerical characters (no. 1-6 wells) in white or red. Red means that the ion signal was thought to have been detected. 


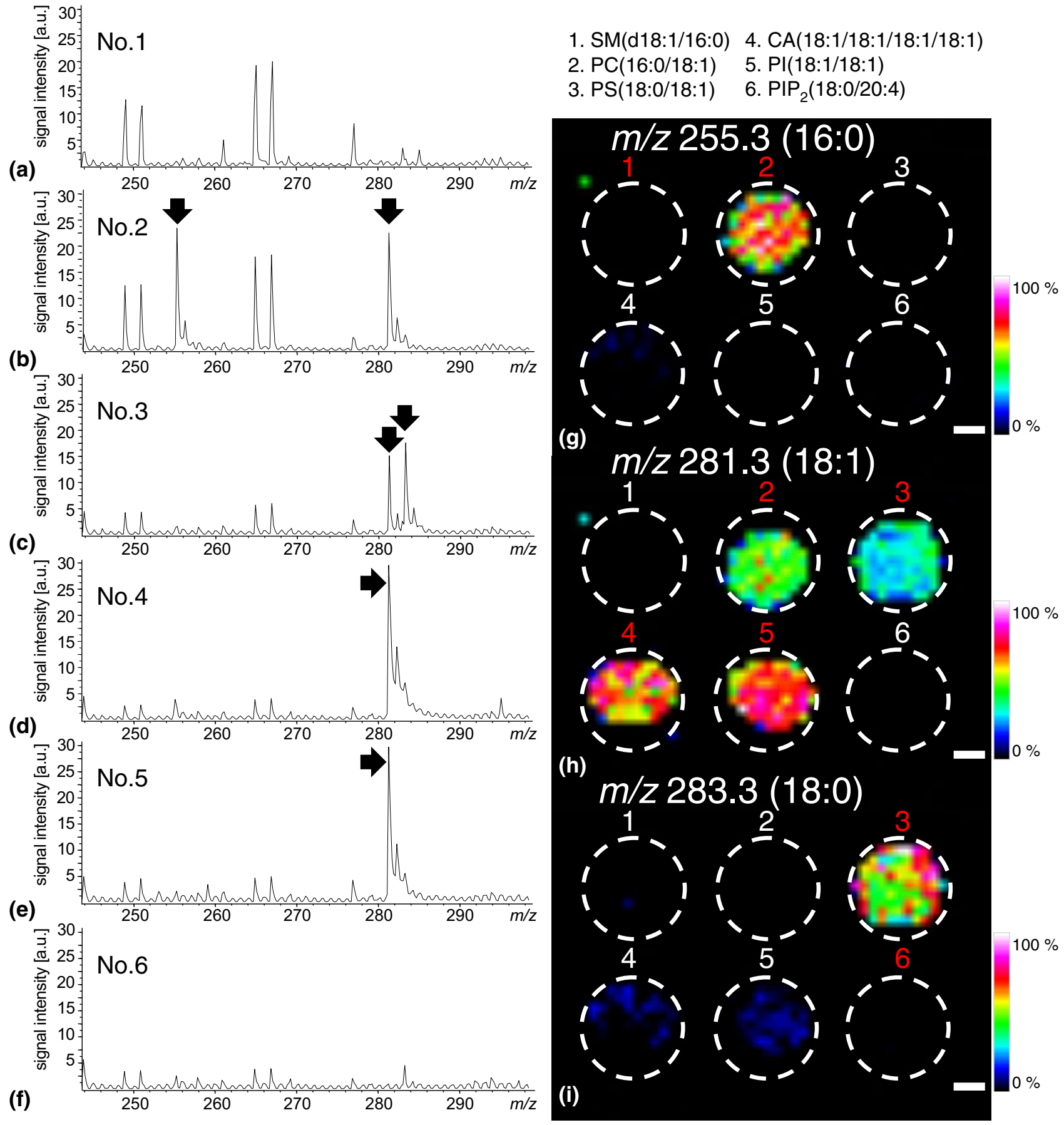

Figure 3. Identification of fatty acids from standard lipids in nano-PALDI-IMS. The standard lipids, such as SM (d18:1/16:0) (no. 1 well), PC (16:0/18:1) (no. 2 well), PS (18:0/18:1) (no. 3 well), CA (18:1/18:1/18:1/18:1) (no. 4 well), PI (18:1/18:1) (no. 5 well), and PIP $_{2}(18: 0 / 20: 4)$ (no. 6 well), were deposited by $1 \mathrm{nmol}$ on the each well of the target plate. The surface was measured with the scan pitch of $300 \mu \mathrm{m}$ by nano-PALDI-IMS analysis in negative-ion mode. The representative mass spectra in each well are shown: no.1 (a), 2 (b), 3 (c), 4 (d), 5 (e), and 6 (f). The peaks used to reconstruct ion image are indicated by arrows. The ion images at $\mathrm{m} / \mathrm{z} 255.3(\mathrm{~g}), \mathrm{m} / \mathrm{z} 281.3$ (h), and $\mathrm{m} / \mathrm{z} 283.3$ (i) were reconstructed. The signals correspond to palmitic acid (16:0), oleic acid (18:1), and stearic acid (18:0), respectively. The numerical characters in red mean that the ion signal was thought to have been detected. The positions of each well are circled with white dot lines. The scale bars are $1 \mathrm{~mm}$.

An ion image of palmitic acid $(m / z 255.3)$ was observed at the no. 2 well, which contained PC (16:0/18:1), but no ion signal was seen at the no. 1 well, which contained SM (d18:1/16:0) (Figure 3g). The results obtained from the no. 2 well suggested that the fatty acid was dissociated from PC. However, in the case of the no. 1 well, no ion signal was obtained, possibly due to the difficulty of dissociating fatty acid from the ceramide structure of SM. 
The ion image of oleic acid $(m / z 281.3)$ was observed at wells 2, 3, 4, and 5, which contained PC (16:0/18:1), PS (18:0/18:1), CA (18:1/18:1/18:1/18:1), and PI (18:1/ 18:1), respectively (Figure 3h). The ion images of wells 2 and 3 showed almost the same signal intensities because each oleic acid was dissociated from PC (16:0/ 18:1) and PS (18:0/18:1). The signal intensities of oleic acid at wells 4 and 5 were similar to each other, but the number of oleic acids in CA (18:1/18:1/18:1/18:1) was twice that in PI (18:1/18:1). This result also suggested that the dissociation effect from the lipids might be due to the structures of the lipids.

The ion image of stearic acid $(\mathrm{m} / \mathrm{z} 283.3)$ was observed at well 3, which was composed of PS (18:0/18:1) (Figure 3i). A weak ion signal of stearic acid $(\mathrm{m} / \mathrm{z} 283.3)$ was observed at wells 4 and 5, possibly due to the contaminations of the isotope series peaks relevant to a monoisotopic peak of $m / z 281.3$ as shown in Figure $3 \mathrm{~d}$ and e. On the other hand, the ion signal of stearic acid was not observed at well 6, which contained $\mathrm{PIP}_{2}$ (18:0/20:4), possibly due to the fragmentation of molecules from the complex structure of this lipid. This result suggested that the fatty acids could be detected by the dissociation from several lipids as well as by the free fatty acid in tissue sections. We expect that AgNPs could be successfully applied to the analysis of fatty acids in biological tissue sections.
Our previous study reported that nano-PALDI-IMS using $\mathrm{Fe}_{2} \mathrm{O}_{3} \mathrm{NPs}$ detected PC, galactosylceramide, sulfatide, and phosphatidylserine as major peaks in mouse brain tissues $[17,18]$. This method might also be used to detect fatty acids as minor peaks in negative-ion mode. In the present study, we detected fatty acids as major peaks in nano-PALDI-IMS using AgNPs. The mechanism by which fatty acids are ionized using AgNPs is unknown. However, we have evidence that two other AgNPs capped by single organic component, myristate or stearylamine, also contributed to the detection of fatty acids in biological tissue (data not shown). So, fatty acids composed of AgNPs might not be dependent on the ionization of fatty acids from lipids, and Ag core would have the specificity to ionize fatty acids in nano-PALDI-IMS.

\section{Identification of Fatty Acids in Mouse Retinal Sections in nano-PALDI-IMS}

The mouse retina is found to be suitable for nanoPALDI-IMS, possibly due to its complex structure, which includes eight different layers. In the present experiment, mouse retinal sections were analyzed at a high spatial resolution with a scan pitch of $10 \mu \mathrm{m}$ (gray area in Figure 4a), and then the ion images corresponding to seven fatty acids were reconstructed (Figure
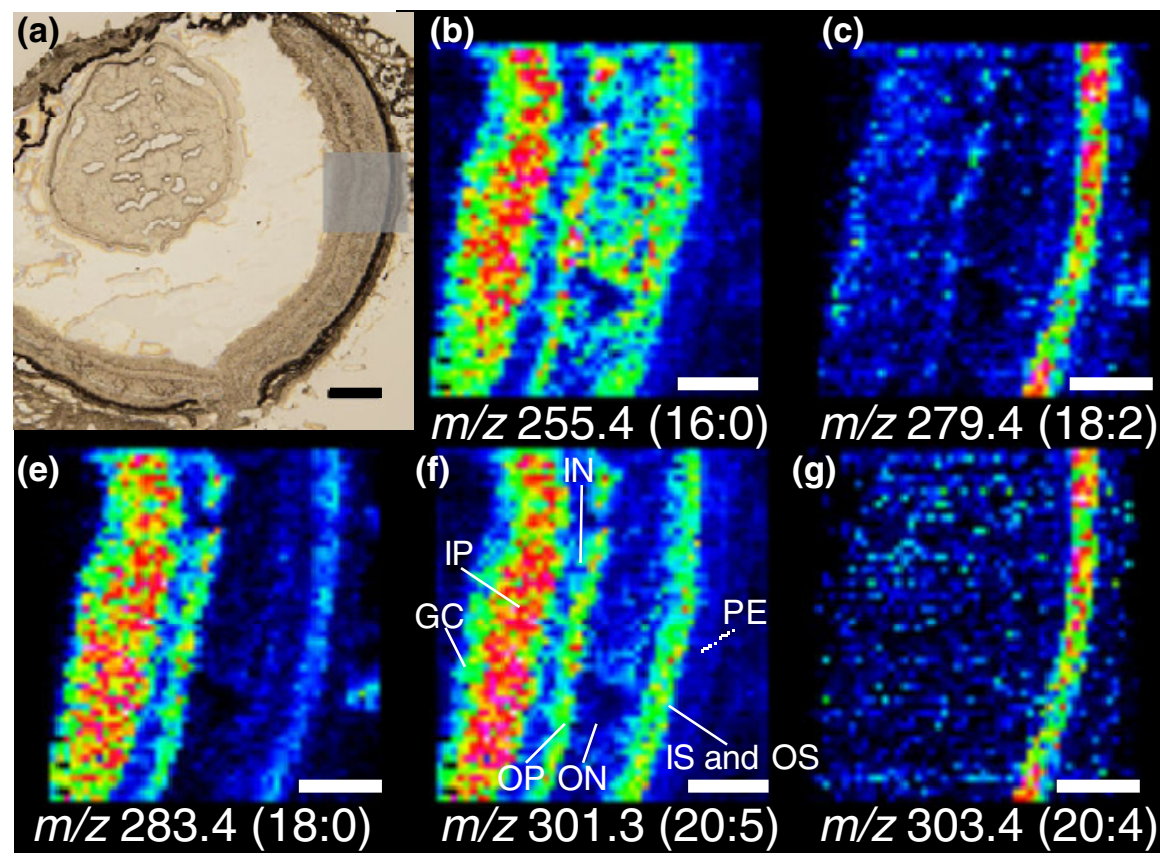

(f)

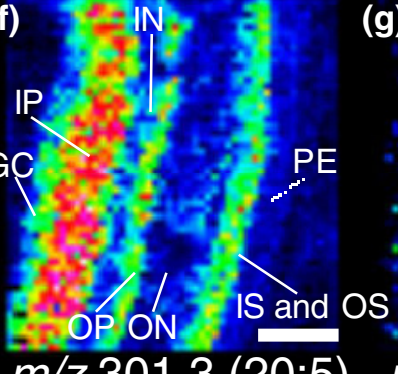

$m / z 279.4(18: 2)$
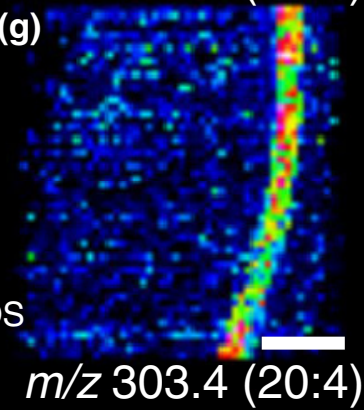

(d).

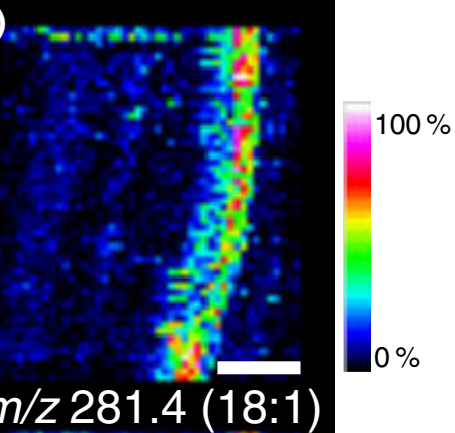

Figure 4. Identification of fatty acids in mouse retinal sections in nano-PALDI-IMS. The mouse retinal section was sliced to a thickness of $10 \mu \mathrm{m}$ (a). The measurement area is covered with gray area. The black scale bar is $200 \mu \mathrm{m}$. After spraying with AgNPs solution, the surface of retinal section was measured with a scan pitch of $10 \mu \mathrm{m}$ by nano-PALDI-IMS analysis in negative-ion mode. The ion images were reconstructed from the peaks corresponding to fatty acids, such as palmitic acid (16:0, $\mathrm{m} / \mathrm{z}$ 255.4) (b), linoleic acid (18:2, $m / z$ 279.4) (c), oleic acid $(18: 1, m / z 281.4)($ d), stearic acid $(18: 0, m / z 283.4)$ (e), EPA (20:5, m/z 301.3), (f), AA (20:4, m/z 303.4) (g), and DHA (22:6, $m / z$ 327.3) (h). The white scale bars are $100 \mu \mathrm{m}$. 
$4 b-h)$. The ion image of EPA (20:5) showed the distinct layered structure of the mouse retina. The layers are named as follows: ganglion cell layer (GC), inner plexiform layer (IP), inner nuclear layer (IN), outer plexiform layer $(\mathrm{OP})$, outer nuclear layer $(\mathrm{ON})$, inner segment (IS), outer segment (OS), and pigment epithelium (PE) from the side of the vitreous body.

Our previous study reported that PC has three-zonal distribution in mouse retinal sections and that the distributions were divided according to the difference in fatty acids composition in PC [2]. The spatial resolution depends on the diameter of the laser equipped with a mass spectrometer. The ion images reconstructed in nano-PALDI-IMS with the use of AgNPs showed obviously six-zonal distributions of fatty acids. The ion image showed that palmitic acid (16:0) was distributed in all regions of the mouse retina except the PE region (Figure 4b). Linoleic acid (18:2, Figure 4c), oleic acid (18:1, Figure 4d), and AA (20:4, Figure 4g) were equally distributed at PE. Stearic acid (18:0) was found at GC, $\mathrm{IP}, \mathrm{OP}$, and PE regions of the retina (Figure 4e). However, the ion image of EPA (20:5, Figure 4f) was similar to the ion image of palmitic acid (Figure $4 \mathrm{~b}$ ). The ion image of DHA was also slightly apparent at the GC, IP, and $\mathrm{OP}$ regions except at the PE region, where the ion signal was detected at high intensity (Figure 4h). Their observed fatty acids might be free fatty acids, or they might be decomposition from other lipids based on the earlier findings.

Our previous study revealed that PC composed of DHA was strongly distributed around the PE [2]. In the present experiment, our results revealed that DHA was distributed at the PE in mouse retina. DHA plays an important role in the development of visual function [23], in promoting survival [29], and in inhibiting apoptosis of photoreceptors [30]. In addition, LCPUFAs including DHA are a major target of peroxidation, which contributes to several diseases, such as agerelated macular degeneration [31], diabetic retinopathy [32], and Smith-Lemli-Opitz syndrome [33]. The use of AgNPs in nano-PALDI-IMS might be helpful for learning the distributions of LCPUFA to elucidate their pathogenesis. Thus, our results indicated that AgNPs were successfully applied to nano-PALDI-IMS analysis of fatty acids in biological samples with heterogeneous structures, such as mouse retinal sections. NanoPALDI-IMS using AgNPs is expected to be useful for analyzing basic biological mechanisms and several diseases through the distribution of fatty acids.

\section{Conclusions}

The present study successfully demonstrated the visualization of fatty acids in mouse liver and retinal tissue samples by using AgNPs in nano-PALDI-IMS. In the mouse retinal sections, the ion images from seven kinds of fatty acids indicated the six-zonal distribution, as supported by our previous study. The use of AgNPs for nano-PALDI-IMS analysis is expected to help eluci- date several diseases in biological tissues as well as the basic biological mechanisms, and thus to help prevent disease.

\section{Acknowledgments}

The authors are grateful to Yukiko Sugiyama (Hamamatsu University School of Medicine) for providing considerable support to our research. The authors acknowledge support for this work by a Grant-in-Aid for SENTAN from the Japan Science and Technology Agency (to M.S.) and by a Grant-in-Aid for Young Scientists S (to M.S.) and Young Scientists B (to T.H.).

\section{References}

1. Shimma, S.; Sugiura, Y.; Hayasaka, T.; Zaima, N.; Matsumoto, M.; Setou, M. Mass Imaging and Identification of Biomolecules with MALDI-QIT-TOF-Based System. Anal. Chem. 2008, 80(3), 878-885.

2. Hayasaka, T.; Goto-Inoue, N.; Sugiura, Y.; Zaima, N.; Nakanishi, H. Ohishi, K.; Nakanishi, S.; Naito, T.; Taguchi, R.; Setou, M. MatrixAssisted Laser Desorption/Ionization Quadrupole Ion Trap Time-ofFlight (MALDI-QIT-TOF)-Based Imaging Mass Spectrometry Reveals a Layered Distribution of Phospholipid Molecular Species in the Mouse Retina. Rapid Commun. Mass Spectrom. 2008, 22(21), 3415-3426.

3. Hayasaka, T.; Goto-Inoue, N.; Zaima, N.; Kimura, Y.; Setou, M. OrganSpecific Distributions of Lysophosphatidylcholine and Triacylglycerol in Mouse Embryo. Lipids 2009, 44(9), 837-848.

4. Zaima, N.; Hayasaka, T.; Goto-Inoue, N.; Setou, M. Imaging of Metabolites by MALDI Mass Spectrometry. J. Oleo. Sci. 2009, 58(8), 415-419.

5. Groseclose, M. R.; Andersson, M.; Hardesty, W. M.; Caprioli, R. M. Identification of Proteins Directly from Tissue: In Situ Tryptic Digestions Coupled with Imaging Mass Spectrometry. J. Mass Spectrom. 2007, 42(2), 254-262.

6. Andersson, M.; Groseclose, M. R.; Deutch, A. Y.; Caprioli, R. M. Imaging Mass Spectrometry of Proteins and Peptides: 3D Volume Reconstruction. Nat. Methods 2008, 5(1), 101-108.

7. Goto-Inoue, N.; Hayasaka, T.; Sugiura, Y.; Taki, T.; Li, Y. T.; Matsumoto, M.; Setou, M. High-Sensitivity Analysis of Glycosphingolipids by Matrix-Assisted Laser Desorption/Ionization Quadrupole Ion Trap Time-of-Flight Imaging Mass Spectrometry on Transfer Membranes. J. Chromatogr. B Analyt. Technol. Biomed. Life Sci. 2008, 870(1), 74-83.

8. Goto-Inoue, N.; Hayasaka, T.; Zaima, N.; Setou, M. The Specific Localization of Seminolipid Molecular Species on Mouse Testis During Testicular Maturation Revealed by Imaging Mass Spectrometry. Glycobiology 2009, 19(9), 950-957.

9. Shimma, S.; Sugiura, Y.; Hayasaka, T.; Hoshikawa, Y.; Noda, T.; Setou, M. MALDI-Based Imaging Mass Spectrometry Revealed Abnormal Distribution of Phospholipids in Colon Cancer Liver Metastasis. J. Chromatogr. B Analyt. Technol. Biomed. Life Sci. 2007, 855(1), 98-103.

10. Wen, X.; Dagan, S.; Wysocki, V. H. Small-Molecule Analysis with Silicon-Nanoparticle-Assisted Laser Desorption/Ionization Mass Spectrometry. Anal. Chem. 2007, 79(2), 434-444.

11. Wu, H. P.; Yu, C. J.; Lin, C. Y.; Lin, Y. H.; Tseng, W. L. Gold Nanoparticles as Assisted Matrices for the Detection of Biomolecules in a High-Salt Solution Through Laser Desorption/Ionization Mass Spectrometry. J. Am. Soc. Mass Spectrom. 2009, 20(5), 875-882.

12. Shrivas, K.; Wu, H. F. Applications of Silver Nanoparticles Capped with Different Functional Groups as the Matrix and Affinity Probes in Surface-Assisted Laser Desorption/Ionization Time-of-Flight and Atmospheric Pressure Matrix-Assisted Laser Desorption/Ionization Ion Trap Mass Spectrometry for Rapid Analysis of Sulfur Drugs and Biothiols in Human Urine. Rapid Commun. Mass Spectrom. 2008, 22(18), 2863-2872.

13. Chiu, T. C.; Chang, L. C.; Chiang, C. K.; Chang, H. T. Determining Estrogens Using Surface-Assisted Laser Desorption/Ionization Mass Spectrometry with Silver Nanoparticles as the Matrix. J. Am. Soc. Mass Spectrom. 2008, 19(9), 1343-1346.

14. Sherrod, S. D.; Diaz, A. J.; Russell, W. K.; Cremer, P. S.; Russell, D. H. Silver Nanoparticles as Selective Ionization Probes for Analysis of Olefins by Mass Spectrometry. Anal. Chem. 2008, 80(17), 6796-6799.

15. Watanabe, T.; Okumura, K.; Kawasaki, H.; Arakawa, R. Effect of Urea Surface Modification and Photocatalytic Cleaning on Surface-Assisted Laser Desorption Ionization Mass Spectrometry with Amorphous TiO2 Nanoparticles. J. Mass Spectrom. 2009, 44(10), 1443-1451.

16. Wang, M. T.; Liu, M. H.; Wang, C. R.; Chang, S. Y. Silver-Coated Gold Nanoparticles as Concentrating Probes and Matrices for Surface-Assisted Laser Desorption/Ionization Mass Spectrometric Analysis of Aminoglycosides. J. Am. Soc. Mass Spectrom. 2009, 20(10), 1925-1932.

17. Taira, S.; Sugiura, Y.; Moritake, S.; Shimma, S.; Ichiyanagi, Y.; Setou, M. Nanoparticle-Assisted Laser Desorption/Ionization Based Mass Imaging with Cellular Resolution. Anal. Chem. 2008, 80(12), 4761-4766.

18. Ageta, H.; Asai, S.; Sugiura, Y.; Goto-Inoue, N.; Zaima, N.; Setou, M. Layer-Specific Sulfatide Localization in Rat Hippocampus Middle Molec- 
ular Layer is Revealed by Nanoparticle-Assisted Laser Desorption/ Ionization Imaging Mass Spectrometry. Med. Mol. Morphol. 2009, 42(1), $16-23$.

19. Novak, E. M.; Dyer, R. A.; Innis, S. M. High Dietary Omega-6 Fatty Acids Contribute to Reduced Docosahexaenoic Acid in the Developing Brain and Inhibit Secondary Neurite Growth. Brain Res. 2008, 1237, 136-145.

20. Rousseau, D.; Helies-Toussaint, C.; Raederstorff, D.; Moreau, D.; Grynberg, A. Dietary n-3 Polyunsaturated Fatty Acids Affect the Development of Renovascular Hypertension in Rats. Mol. Cell. Biochem. 2001, 225(1), 109-119.

21. Farkas, K.; Ratchford, I. A.; Noble, R. C.; Speake, B. K. Changes in the Size and Docosahexaenoic Acid Content of Adipocytes During Chick Embryo Development. Lipids 1996, 31(3), 313-321.

22. Innis, S. M.; Friesen, R. W. Essential n-3 Fatty Acids in Pregnant Women and Early Visual Acuity Maturation in Term Infants. Am. J. Clin. Nutr. 2008, 87(3), 548-557.

23. Alessandri, J. M.; Goustard, B.: Guesnet, P.; Durand, G. Docosahexaenoic Acid Concentrations in Retinal Phospholipids of Piglets Fed an Infant Formula Enriched with Long-Chain Polyunsaturated Fatty Acids: Effects of Egg Phospholipids and Fish Oils with Different Ratios of Eicosapentaenoic Acid to Docosahexaenoic Acid. Am. J. Clin. Nutr. 1998, 67(3), 377-385.

24. Innis, S. M. Essential Fatty Acids in Growth and Development. Prog. Lipid Res. 1991, 30(1), 39-103.

25. Kennedy, A.; Martinez, K.; Chuang, C. C.; LaPoint, K.; McIntosh, M. Saturated Fatty Acid-Mediated Inflammation and Insulin Resistance in Adipose Tissue: Mechanisms of Action and Implications. J. Nutr. 2009, 139(1), 1-4.

26. Yu, H.; Lopez, E.; Young, S. W.; Luo, J.; Tian, H.; Cao, P. Quantitative Analysis of Free Fatty Acids in Rat Plasma Using Matrix-Assisted Laser
Desorption/Ionization Time-of-Flight Mass Spectrometry with Meso-Tetrakis Porphyrin as Matrix. Anal. Biochem. 2006, 354(2), 182-191.

27. Ayorinde, F. O.; Garvin, K.; Saeed, K. Determination of the Fatty Acid Composition of Saponified Vegetable Oils Using Matrix-Assisted Laser Desorption/Ionization Time-of-Flight Mass Spectrometry. Rapid Commun. Mass Spectrom. 2000, 14(7), 608-615.

28. Kashiwagi, Y.; Yamamoto, M.; Nakamoto, M. Facile Size-Regulated Synthesis of Silver Nanoparticles by Controlled Thermolysis of Silver Alkylcarboxylates in the Presence of Alkylamines with Different Chain Lengths. J. Colloid Interface Sci. 2006, 300(1), 169-175.

29. Rotstein, N. P.; Aveldano, M. I.; Barrantes, F. J.; Politi, L. E. Docosahexaenoic Acid is Required for the Survival of Rat Retinal Photoreceptors In Vitro. J. Neurochem. 1996, 66(5), 1851-1859.

30. Rotstein, N. P.; Aveldano, M. I.; Barrantes, F. J.; Roccamo, A. M.; Politi, L. E. Apoptosis of Retinal Photoreceptors During Development In Vitro: protective effect of docosahexaenoic acid. J. Neurochem. 1997, 69(2), 504-513.

31. Suzuki, M.; Kamei, M.; Itabe, H.; Yoneda, K.; Bando, H.; Kume, N. Tano, Y. Oxidized Phospholipids in the Macula Increase with Age and in Eyes with Age-Related Macular Degeneration. Mol. Vis. 2007, 13 772-778.

32. Pan, H. Z.; Zhang, H.; Chang, D.; Li, H.; Sui, H. The Change of Oxidative Stress Products in Diabetes Mellitus and Diabetic Retinopathy. Br. J. Ophthalmol. 2008, 92(4), 548-551.

33. Ford, D. A.; Monda, J. K.; Brush, R. S.; Anderson, R. E.; Richards, M. J.; Fliesler, S. J. Lipidomic Analysis of the Retina in a Rat Model of Smith-Lemli-Opitz Syndrome: Alterations in Docosahexaenoic Acid Content of Phospholipid Molecular Species. J. Neurochem. 2008, 105(3), 1032-1047. 